\author{
S. S. Dragomir
}

\title{
SOME RESULTS FOR ISOTONIC FUNCTIONALS VIA AN INEQUALITY DUE TO KITTANEH AND MANASRAH
}

\begin{abstract}
In this paper we obtain some inequalities for isotonic functionals via a reverse and refinement of Young's inequality due to Kittaneh and Manasrah.

KEY WORDS: isotonic functionals, Hölder's inequality, Schwarz's inequality, Callebaut's inequality, integral inequalities, discrete inequalities.
\end{abstract}

AMS Mathematics Subject Classification: 26D15, 26D10.

\section{Introduction}

Let $L$ be a linear class of real-valued functions $g: E \rightarrow \mathbb{R}$ having the properties

(L1) $f, g \in L$ imply $(\alpha f+\beta g) \in L$ for all $\alpha, \beta \in \mathbb{R}$;

(L2) $\mathbf{1} \in L$, i.e., if $f_{0}(t)=1, t \in E$ then $f_{0} \in L$.

An isotonic linear functional $A: L \rightarrow \mathbb{R}$ is a functional satisfying

(A1) $A(\alpha f+\beta g)=\alpha A(f)+\beta A(g)$ for all $f, g \in L$ and $\alpha, \beta \in \mathbb{R}$.

(A2) If $f \in L$ and $f \geq 0$, then $A(f) \geq 0$.

The mapping $A$ is said to be normalised if

(A3) $A(\mathbf{1})=1$.

Isotonic, that is, order-preserving, linear functionals are natural objects in analysis which enjoy a number of convenient properties. Thus, they provide, for example, Jessen's inequality, which is a functional form of Jensen's inequality (see [2], [15] and [16]). For other inequalities for isotonic functionals see [1], [4]-[14] and [17]-[20].

We note that common examples of such isotonic linear functionals $A$ are given by

$$
A(g)=\int_{E} g d \mu \text { or } A(g)=\sum_{k \in E} p_{k} g_{k}
$$


where $\mu$ is a positive measure on $E$ in the first case and $E$ is a subset of the natural numbers $\mathbb{N}$, in the second $\left(p_{k} \geq 0, k \in E\right)$.

As is known to all, the famous Young inequality for scalars says that if $a, b>0$ and $\nu \in[0,1]$, then

$$
a^{1-\nu} b^{\nu} \leq(1-\nu) a+\nu b
$$

with equality if and only if $a=b$. The inequality (1) is also called $\nu$-weighted arithmetic-geometric mean inequality.

Kittaneh and Manasrah [12], [13] provided a refinement and a reverse for Young inequality as follows:

$$
r(\sqrt{a}-\sqrt{b})^{2} \leq(1-\nu) a+\nu b-a^{1-\nu} b^{\nu} \leq R(\sqrt{a}-\sqrt{b})^{2}
$$

where $a, b>0, \nu \in[0,1], r=\min \{1-\nu, \nu\}$ and $R=\max \{1-\nu, \nu\}$. The case $\nu=\frac{1}{2}$ reduces (2) to an identity and is of no interest.

We observe that, if $a, b \in[m, M] \subset(0, \infty)$, then $|\sqrt{a}-\sqrt{b}| \leq \sqrt{M}-\sqrt{m}$ and by (2) we obtain the following reverse of Young inequality

$$
(1-\nu) a+\nu b-a^{1-\nu} b^{\nu} \leq R(\sqrt{M}-\sqrt{m})^{2} .
$$

We can give a simple direct proof for (2) as follows.

Recall the following result obtained by Dragomir in 2006 [8] that provides a refinement and a reverse for the weighted Jensen's discrete inequality:

$$
\begin{aligned}
& \min _{j \in\{1,2, \ldots, n\}}\left\{p_{j}\right\}\left[\frac{1}{n} \sum_{j=1}^{n} \Phi\left(x_{j}\right)-\Phi\left(\frac{1}{n} \sum_{j=1}^{n} x_{j}\right)\right] \\
& \quad \leq \frac{1}{P_{n}} \sum_{j=1}^{n} p_{j} \Phi\left(x_{j}\right)-\Phi\left(\frac{1}{P_{n}} \sum_{j=1}^{n} p_{j} x_{j}\right) \\
& \quad \leq n \max _{j \in\{1,2, \ldots, n\}}\left\{p_{j}\right\}\left[\frac{1}{n} \sum_{j=1}^{n} \Phi\left(x_{j}\right)-\Phi\left(\frac{1}{n} \sum_{j=1}^{n} x_{j}\right)\right],
\end{aligned}
$$

where $\Phi: C \rightarrow \mathbb{R}$ is a convex function defined on convex subset $C$ of the linear space $X,\left\{x_{j}\right\}_{j \in\{1,2, \ldots, n\}}$ are vectors in $C$ and $\left\{p_{j}\right\}_{j \in\{1,2, \ldots, n\}}$ are nonnegative numbers with $P_{n}=\sum_{j=1}^{n} p_{j}>0$.

For $n=2$, we deduce from (4) that

$$
\begin{aligned}
& 2 \min \{\nu, 1-\nu\}\left[\frac{\Phi(x)+\Phi(y)}{2}-\Phi\left(\frac{x+y}{2}\right)\right] \\
& \quad \leq \nu \Phi(x)+(1-\nu) \Phi(y)-\Phi[\nu x+(1-\nu) y] \\
& \quad \leq 2 \max \{\nu, 1-\nu\}\left[\frac{\Phi(x)+\Phi(y)}{2}-\Phi\left(\frac{x+y}{2}\right)\right]
\end{aligned}
$$


for any $x, y \in \mathbb{R}$ and $\nu \in[0,1]$.

If we take $\Phi(x)=\exp (x)$, then we get from (5)

$$
\begin{aligned}
& 2 \min \{\nu, 1-\nu\}\left[\frac{\exp (x)+\exp (y)}{2}-\exp \left(\frac{x+y}{2}\right)\right] \\
& \quad \leq \nu \exp (x)+(1-\nu) \exp (y)-\exp [\nu x+(1-\nu) y] \\
& \quad \leq 2 \max \{\nu, 1-\nu\}\left[\frac{\exp (x)+\exp (y)}{2}-\exp \left(\frac{x+y}{2}\right)\right]
\end{aligned}
$$

for any $x, y \in \mathbb{R}$ and $\nu \in[0,1]$. Further, denote $\exp (x)=a, \exp (y)=b$ with $a, b>0$, then from (6) we obtain the inequality (2).

In this paper we obtain some inequalities for isotonic functionals via the reverse and refinement of Young's inequality (2). Applications for integrals and $n$-tuples of real numbers are also provided.

\section{On Callebaut's inequality}

The functional version of Callebaut inequality states that

$$
A^{2}(f g) \leq A\left(f^{2-\nu} g^{\nu}\right) A\left(f^{\nu} g^{2-\nu}\right) \leq A\left(f^{2}\right) A\left(g^{2}\right)
$$

provided that $f^{2}, g^{2}, f^{2-\nu} g^{\nu}, f^{\nu} g^{2-\nu}, f g \in L$ for some $\nu \in[0,2]$. For the discrete and integral of one real variable versions see [3].

We have the following result that provides a refinement and reverse of Callebaut's inequality:

Theorem 1. Let $A, B: L \rightarrow \mathbb{R}$ be two normalised isotonic functionals. If $f, g: E \rightarrow \mathbb{R}$ are such that, $f^{2}, g^{2}, f g, f^{2(1-\nu)} g^{2 \nu}, f^{2 \nu} g^{2(1-\nu)} \in L$ for some $\nu \in[0,1]$, then

$$
\begin{aligned}
r( & \left.A\left(f^{2}\right) B\left(g^{2}\right)-2 A(f g) B(f g)+A\left(g^{2}\right) B\left(f^{2}\right)\right) \\
\leq & (1-\nu) A\left(f^{2}\right) B\left(g^{2}\right)+\nu A\left(g^{2}\right) B\left(f^{2}\right) \\
& -A\left(f^{2(1-\nu)} g^{2 \nu}\right) B\left(f^{2 \nu} g^{2(1-\nu)}\right) \\
& \leq R\left(A\left(f^{2}\right) B\left(g^{2}\right)-2 A(f g) B(f g)+A\left(g^{2}\right) B\left(f^{2}\right)\right),
\end{aligned}
$$

where $r=\min \{1-\nu, \nu\}$ and $R=\max \{1-\nu, \nu\}$.

Proof. Let $x, y \in E$ such that $g(x), g(y) \neq 0$. If we use the inequalities (2) for

$$
a=\frac{f^{2}(x)}{g^{2}(x)}, \quad b=\frac{f^{2}(y)}{g^{2}(y)}
$$


then we get

$$
\begin{aligned}
& r\left(\frac{f(x)}{g(x)}-\frac{f(y)}{g(y)}\right)^{2} \\
& \quad \leq(1-\nu) \frac{f^{2}(x)}{g^{2}(x)}+\nu \frac{f^{2}(y)}{g^{2}(y)}-\left(\frac{f^{2}(x)}{g^{2}(x)}\right)^{1-\nu}\left(\frac{f^{2}(y)}{g^{2}(y)}\right)^{\nu} \\
& \quad \leq R\left(\frac{f(x)}{g(x)}-\frac{f(y)}{g(y)}\right)^{2}
\end{aligned}
$$

where $\nu \in[0,1], r=\min \{1-\nu, \nu\}$ and $R=\max \{1-\nu, \nu\}$.

Therefore

$$
\begin{aligned}
& r\left(\frac{f^{2}(x)}{g^{2}(x)}-2 \frac{f(x)}{g(x)} \frac{f(y)}{g(y)}+\frac{f^{2}(y)}{g^{2}(y)}\right) \\
& \quad \leq(1-\nu) \frac{f^{2}(x)}{g^{2}(x)}+\nu \frac{f^{2}(y)}{g^{2}(y)}-\left(\frac{f^{2}(x)}{g^{2}(x)}\right)^{1-\nu}\left(\frac{f^{2}(y)}{g^{2}(y)}\right)^{\nu} \\
& \quad \leq R\left(\frac{f^{2}(x)}{g^{2}(x)}-2 \frac{f(x)}{g(x)} \frac{f(y)}{g(y)}+\frac{f^{2}(y)}{g^{2}(y)}\right) .
\end{aligned}
$$

If we multiply (10) by $g^{2}(x) g^{2}(y)$, then we get

$$
\begin{aligned}
r( & \left.f^{2}(x) g^{2}(y)-2 f(x) g(x) f(y) g(y)+f^{2}(y) g^{2}(x)\right) \\
\leq & (1-\nu) f^{2}(x) g^{2}(y)+\nu g^{2}(x) f^{2}(y) \\
& -f^{2(1-\nu)}(x) g^{2 \nu}(x) f^{2 \nu}(y) g^{2(1-\nu)}(y) \\
& \leq R\left(f^{2}(x) g^{2}(y)-2 f(x) g(x) f(y) g(y)+f^{2}(y) g^{2}(x)\right),
\end{aligned}
$$

which holds for any $x, y \in E$.

Fix $y \in E$. Then by (11) we have in the order of $L$ that

$$
\begin{aligned}
& r\left(g^{2}(y) f^{2}-2 f(y) g(y) f g+f^{2}(y) g^{2}\right) \\
& \quad \leq(1-\nu) g^{2}(y) f^{2}+\nu f^{2}(y) g^{2}-f^{2 \nu}(y) g^{2(1-\nu)}(y) f^{2(1-\nu)} g^{2 \nu} \\
& \quad \leq R\left(g^{2}(y) f^{2}-2 f(y) g(y) f g+f^{2}(y) g^{2}\right) .
\end{aligned}
$$

If we take the functional $A$ in (12), then we get

$$
\begin{aligned}
& r\left(g^{2}(y) A\left(f^{2}\right)-2 f(y) g(y) A(f g)+f^{2}(y) A\left(g^{2}\right)\right) \\
& \leq(1-\nu) g^{2}(y) A\left(f^{2}\right)+\nu f^{2}(y) A\left(g^{2}\right) \\
& \quad-f^{2 \nu}(y) g^{2(1-\nu)}(y) A\left(f^{2(1-\nu)} g^{2 \nu}\right) \\
& \leq R\left(g^{2}(y) A\left(f^{2}\right)-2 f(y) g(y) A(f g)+f^{2}(y) A\left(g^{2}\right)\right),
\end{aligned}
$$


for any $y \in E$.

If we write this inequality in the order of $L$, then we have

$$
\begin{aligned}
& r\left(A\left(f^{2}\right) g^{2}-2 A(f g) f g+A\left(g^{2}\right) f^{2}\right) \\
& \quad \leq(1-\nu) A\left(f^{2}\right) g^{2}+\nu A\left(g^{2}\right) f^{2}-A\left(f^{2(1-\nu)} g^{2 \nu}\right) f^{2 \nu} g^{2(1-\nu)} \\
& \quad \leq \operatorname{Rr}\left(A\left(f^{2}\right) g^{2}-2 A(f g) f g+A\left(g^{2}\right) f^{2}\right),
\end{aligned}
$$

and by taking the functional $B$ we deduce the desired result (8).

Corollary 1. Let $A: L \rightarrow \mathbb{R}$ be two normalised isotonic functionals. If $f, g: E \rightarrow \mathbb{R}$ are such that $f^{2}, g^{2}, f g, f^{2(1-\nu)} g^{2 \nu}, f^{2 \nu} g^{2(1-\nu)} \in L$ for some $\nu \in[0,1]$, then

$$
\begin{aligned}
2 r & \left(A\left(f^{2}\right) A\left(g^{2}\right)-A^{2}(f g)\right) \\
& \leq A\left(f^{2}\right) A\left(g^{2}\right)-A\left(f^{2(1-\nu)} g^{2 \nu}\right) A\left(f^{2 \nu} g^{2(1-\nu)}\right) \\
& \leq 2 R\left(A\left(f^{2}\right) A\left(g^{2}\right)-A^{2}(f g)\right) .
\end{aligned}
$$

The following result also holds:

Theorem 2. Let $A, B: L \rightarrow \mathbb{R}$ be two normalised isotonic functionals. If $f, g: E \rightarrow \mathbb{R}$ are such that $f \geq 0, g>0, f^{2}, g^{2}, f^{2(1-\nu)} g^{2 \nu}, f^{2 \nu} g^{2(1-\nu)} \in L$ for some $\nu \in[0,1]$ and

$$
0<m \leq \frac{f}{g} \leq M<\infty
$$

for some constants $m, M$, then

$$
\begin{gathered}
(1-\nu) A\left(f^{2}\right) B\left(g^{2}\right)+\nu A\left(g^{2}\right) B\left(f^{2}\right) \\
\quad-A\left(f^{2(1-\nu)} g^{2 \nu}\right) B\left(f^{2 \nu} g^{2(1-\nu)}\right) \\
\leq R(M-m)^{2} A\left(g^{2}\right) B\left(g^{2}\right) .
\end{gathered}
$$

In particular, we have

$$
\begin{aligned}
& A\left(f^{2}\right) A\left(g^{2}\right)-A\left(f^{2(1-\nu)} g^{2 \nu}\right) A\left(f^{2 \nu} g^{2(1-\nu)}\right) \\
& \quad \leq R(M-m)^{2} A^{2}\left(g^{2}\right) .
\end{aligned}
$$

Proof. For any $x, y \in E$ we have

$$
m^{2} \leq \frac{f^{2}(x)}{g^{2}(x)}, \frac{f^{2}(y)}{g^{2}(y)} \leq M^{2} .
$$


If we use the inequality (3) for

$$
a=\frac{f^{2}(x)}{g^{2}(x)}, \quad b=\frac{f^{2}(y)}{g^{2}(y)},
$$

then we get

$$
(1-\nu) \frac{f^{2}(x)}{g^{2}(x)}+\nu \frac{f^{2}(y)}{g^{2}(y)}-\left(\frac{f^{2}(x)}{g^{2}(x)}\right)^{1-\nu}\left(\frac{f^{2}(y)}{g^{2}(y)}\right)^{\nu} \leq R(M-m)^{2}
$$

for any $x, y \in E$.

Now, if we multiply (17) by $g^{2}(x) g^{2}(y)>0$ then we get

$$
\begin{aligned}
& (1-\nu) f^{2}(x) g^{2}(y)+\nu g^{2}(x) f^{2}(y) \\
& \quad-f^{2(1-\nu)}(x) g^{2 \nu}(x) f^{2 \nu}(y) g^{2(1-\nu)}(y) \\
& \leq R(M-m)^{2} g^{2}(x) g^{2}(y)
\end{aligned}
$$

for any $x, y \in E$.

Fix $y \in E$. Then by (18) we have in the order of $L$ that

$$
\begin{aligned}
& (1-\nu) g^{2}(y) f^{2}+\nu f^{2}(y) g^{2}-f^{2 \nu}(y) g^{2(1-\nu)}(y) f^{2(1-\nu)} g^{2 \nu} \\
& \quad \leq R(M-m)^{2} g^{2}(y) g^{2} .
\end{aligned}
$$

If we take the functional $A$ in (19), then we get

$$
\begin{aligned}
(1-\nu) g^{2}(y) A\left(f^{2}\right)+\nu f^{2}(y) A\left(g^{2}\right) \\
\quad-f^{2 \nu}(y) g^{2(1-\nu)}(y) A\left(f^{2(1-\nu)} g^{2 \nu}\right) \\
\leq R(M-m)^{2} g^{2}(y) A\left(g^{2}\right),
\end{aligned}
$$

for any $y \in E$.

This inequality can be written in the order of $L$ as

$$
\begin{aligned}
& (1-\nu) A\left(f^{2}\right) g^{2}+\nu A\left(g^{2}\right) f^{2}-A\left(f^{2(1-\nu)} g^{2 \nu}\right) f^{2 \nu} g^{2(1-\nu)} \\
& \quad \leq R(M-m)^{2} A\left(g^{2}\right) g^{2} .
\end{aligned}
$$

Now, if we take the functional $B$ in (21), then we get the desired result (15).

Corollary 2. Let $A, B: L \rightarrow \mathbb{R}$ be two normalised isotonic functionals. If $f, g: E \rightarrow \mathbb{R}$ are such that $f \geq 0, g>0, f^{2}, g^{2}, f g \in L$ and the condition (14) is valid, then

$$
\begin{aligned}
& \frac{1}{2}\left[A\left(f^{2}\right) B\left(g^{2}\right)+A\left(g^{2}\right) B\left(f^{2}\right)\right]-A(f g) B(f g) \\
& \quad \leq \frac{1}{2}(M-m)^{2} A\left(g^{2}\right) B\left(g^{2}\right) .
\end{aligned}
$$


In particular, we have

$$
A\left(f^{2}\right) A\left(g^{2}\right)-A^{2}(f g) \leq \frac{1}{2}(M-m)^{2} A^{2}\left(g^{2}\right) .
$$

\section{On Hölder's inequality}

We have:

Theorem 3. Let $A: L \rightarrow \mathbb{R}$ be a normalised isotonic functional and $p, q>1$ with $\frac{1}{p}+\frac{1}{q}=1$. If $f, g: E \rightarrow \mathbb{R}$ are such that $f, g \geq 0$ and $f g, f^{p}$, $g^{q} \in L$ then

$$
\begin{aligned}
2 s & \left(\sqrt{A\left(f^{p}\right) A\left(g^{q}\right)}-A\left(f^{\frac{p}{2}} g^{\frac{q}{2}}\right)\right)\left[A\left(f^{p}\right)\right]^{\frac{1}{p}-\frac{1}{2}}\left[A\left(g^{q}\right)\right]^{\frac{1}{q}-\frac{1}{2}} \\
& \leq\left[A\left(f^{p}\right)\right]^{1 / p}\left[A\left(g^{q}\right)\right]^{1 / q}-A(f g) \\
& \leq 2 S\left(\sqrt{A\left(f^{p}\right) A\left(g^{q}\right)}-A\left(f^{\frac{p}{2}} g^{\frac{q}{2}}\right)\right)\left[A\left(f^{p}\right)\right]^{\frac{1}{p}-\frac{1}{2}}\left[A\left(g^{q}\right)\right]^{\frac{1}{q}-\frac{1}{2}},
\end{aligned}
$$

where $s=\min \left\{\frac{1}{p}, \frac{1}{q}\right\}$ and $S=\max \left\{\frac{1}{p}, \frac{1}{q}\right\}$.

Proof. From (2) we have

$$
s(a+b-2 \sqrt{a b}) \leq \frac{1}{p} a+\frac{1}{q} b-a^{\frac{1}{p}} b^{\frac{1}{q}} \leq S(a+b-2 \sqrt{a b})
$$

where $a, b \geq 0$.

If we choose in (25) $a=\frac{f^{p}}{A\left(f^{p}\right)}, b=\frac{g^{q}}{A\left(g^{q}\right)}$, then we get

$$
\begin{aligned}
& s\left(\frac{f^{p}}{A\left(f^{p}\right)}+\frac{g^{q}}{A\left(g^{q}\right)}-2 \frac{f^{\frac{p}{2}} g^{\frac{q}{2}}}{\sqrt{A\left(f^{p}\right) A\left(g^{q}\right)}}\right) \\
& \quad \leq \frac{1}{p} \frac{f^{p}}{A\left(f^{p}\right)}+\frac{1}{q} \frac{g^{q}}{A\left(g^{q}\right)}-\frac{f g}{\left[A\left(f^{p}\right)\right]^{1 / p}\left[A\left(g^{q}\right)\right]^{1 / q}} \\
& \quad \leq S\left(\frac{f^{p}}{A\left(f^{p}\right)}+\frac{g^{q}}{A\left(g^{q}\right)}-2 \frac{f^{\frac{p}{2}} g^{\frac{q}{2}}}{\sqrt{A\left(f^{p}\right) A\left(g^{q}\right)}}\right)
\end{aligned}
$$

in the order of $L$..

If we take the functional $A$ in (26), then we get

$$
\begin{aligned}
2 s\left(1-\frac{A\left(f^{\frac{p}{2}} g^{\frac{q}{2}}\right)}{\sqrt{A\left(f^{p}\right) A\left(g^{q}\right)}}\right) & \leq 1-\frac{A(f g)}{\left[A\left(f^{p}\right)\right]^{1 / p}\left[A\left(g^{q}\right)\right]^{1 / q}} \\
& \leq 2 S\left(1-\frac{A\left(f^{\frac{p}{2}} g^{\frac{q}{2}}\right)}{\sqrt{A\left(f^{p}\right) A\left(g^{q}\right)}}\right),
\end{aligned}
$$


which is equivalent to the desired result (24).

The following result also holds:

Theorem 4. Let $A: L \rightarrow \mathbb{R}$ be a normalised isotonic functional and $p$, $q>1$ with $\frac{1}{p}+\frac{1}{q}=1$. If $f, g: E \rightarrow \mathbb{R}$ are such that $f g, f^{p}, g^{q} \in L$ and

$$
0<m_{1} \leq f \leq M_{1}<\infty, \quad 0<m_{2} \leq g \leq M_{2}<\infty,
$$

for some constants $m_{1}, m_{2}, M_{1}$ and $M_{2}$, then

$$
\begin{aligned}
0 \leq & {\left[A\left(f^{p}\right)\right]^{1 / p}\left[A\left(g^{q}\right)\right]^{1 / q}-A(f g) } \\
\leq & S\left(\max \left\{\left(\frac{M_{1}}{m_{1}}\right)^{\frac{p}{2}},\left(\frac{M_{2}}{m_{2}}\right)^{\frac{q}{2}}\right\}-\min \left\{\left(\frac{m_{1}}{M_{1}}\right)^{\frac{p}{2}},\left(\frac{m_{2}}{M_{2}}\right)^{\frac{q}{2}}\right\}\right)^{2} \\
& \times\left[A\left(f^{p}\right)\right]^{1 / p}\left[A\left(g^{q}\right)\right]^{1 / q},
\end{aligned}
$$

where $S=\max \left\{\frac{1}{p}, \frac{1}{q}\right\}$.

Proof. Observe that, by (26) we have

$$
m_{1}^{p} \leq A\left(f^{p}\right) \leq M_{1}^{p} \text { and } m_{2}^{q} \leq A\left(g^{q}\right) \leq M_{2}^{q}
$$

Also

$$
\left(\frac{m_{1}}{M_{1}}\right)^{p} \leq \frac{f^{p}}{A\left(f^{p}\right)} \leq\left(\frac{M_{1}}{m_{1}}\right)^{p}
$$

and

$$
\left(\frac{m_{2}}{M_{2}}\right)^{q} \leq \frac{g^{q}}{A\left(g^{q}\right)} \leq\left(\frac{M_{2}}{m_{2}}\right)^{q}
$$

Therefore

$$
\min \left\{\left(\frac{m_{1}}{M_{1}}\right)^{p},\left(\frac{m_{2}}{M_{2}}\right)^{q}\right\} \leq \frac{f^{p}}{A\left(f^{p}\right)}, \frac{g^{q}}{A\left(g^{q}\right)} \leq \max \left\{\left(\frac{M_{1}}{m_{1}}\right)^{p},\left(\frac{M_{2}}{m_{2}}\right)^{q}\right\}
$$

and by (3) we have for $\nu=\frac{1}{q}, a=\frac{f^{p}}{A\left(f^{p}\right)}, b=\frac{g^{q}}{A\left(g^{q}\right)}, m=\min \left\{\left(\frac{m_{1}}{M_{1}}\right)^{p},\left(\frac{m_{2}}{M_{2}}\right)^{q}\right\}$ and $M=\max \left\{\left(\frac{M_{1}}{m_{1}}\right)^{p},\left(\frac{M_{2}}{m_{2}}\right)^{q}\right\}$ that

$$
\text { (29) } \begin{aligned}
\frac{1}{p} & \frac{f^{p}}{A\left(f^{p}\right)}+\frac{1}{q} \frac{g^{q}}{A\left(g^{q}\right)}-\frac{f g}{\left[A\left(f^{p}\right)\right]^{1 / p}\left[A\left(g^{q}\right)\right]^{1 / q}} \\
& \leq S\left(\max \left\{\left(\frac{M_{1}}{m_{1}}\right)^{\frac{p}{2}},\left(\frac{M_{2}}{m_{2}}\right)^{\frac{q}{2}}\right\}-\min \left\{\left(\frac{m_{1}}{M_{1}}\right)^{\frac{p}{2}},\left(\frac{m_{2}}{M_{2}}\right)^{\frac{q}{2}}\right\}\right)^{2} .
\end{aligned}
$$


Now, if we take the functional $A$ in the inequality (29), then we get

$$
\begin{aligned}
1 & -\frac{A(f g)}{\left[A\left(f^{p}\right)\right]^{1 / p}\left[A\left(g^{q}\right)\right]^{1 / q}} \\
& \leq S\left(\max \left\{\left(\frac{M_{1}}{m_{1}}\right)^{\frac{p}{2}},\left(\frac{M_{2}}{m_{2}}\right)^{\frac{q}{2}}\right\}-\min \left\{\left(\frac{m_{1}}{M_{1}}\right)^{\frac{p}{2}},\left(\frac{m_{2}}{M_{2}}\right)^{\frac{q}{2}}\right\}\right)^{2},
\end{aligned}
$$

which is equivalent to the desired result (28).

Corollary 3. Let $A: L \rightarrow \mathbb{R}$ be a normalised isotonic functional. If $f$, $g: E \rightarrow \mathbb{R}$ are such that $f g, f^{2}, g^{2} \in L$ and (27) is satisfied, then

$$
\begin{aligned}
0 \leq & {\left[A\left(f^{2}\right)\right]^{1 / 2}\left[A\left(g^{2}\right)\right]^{1 / 2}-A(f g) } \\
\leq & \frac{1}{2}\left(\max \left\{\frac{M_{1}}{m_{1}}, \frac{M_{2}}{m_{2}}\right\}-\min \left\{\frac{m_{1}}{M_{1}}, \frac{m_{2}}{M_{2}}\right\}\right)^{2} \\
& \times\left[A\left(f^{2}\right)\right]^{1 / 2}\left[A\left(g^{2}\right)\right]^{1 / 2} .
\end{aligned}
$$

\section{Applications for integrals}

Let $(\Omega, \mathcal{A}, \mu)$ be a measurable space consisting of a set $\Omega$, a $\sigma$-algebra $\mathcal{A}$ of parts of $\Omega$ and a countably additive and positive measure $\mu$ on $\mathcal{A}$ with values in $\mathbb{R} \cup\{\infty\}$. For a $\mu$-measurable function $w: \Omega \rightarrow \mathbb{R}$, with $w(x) \geq 0$ for $\mu$-a.e.(almost every) $x \in \Omega$ and $p \geq 1$ consider the Lebesgue space

$$
\begin{aligned}
& L_{w}^{p}(\Omega, \mu):=\{f: \Omega \rightarrow \mathbb{R}, f \text { is } \mu \text {-measurable and } \\
& \left.\qquad \int_{\Omega}|f(x)|^{p} w(x) d \mu(x)<\infty\right\} .
\end{aligned}
$$

For simplicity of notation we write everywhere in the sequel $\int_{\Omega} w d \mu$ instead of $\int_{\Omega} w(x) d \mu(x)$. The same for other integrals involved below. We assume that $\int_{\Omega} w d \mu=1$.

Assume that $f^{2}, g^{2}, f^{2(1-\nu)} g^{2 \nu}, f^{2 \nu} g^{2(1-\nu)} \in L_{w}(\Omega, \mu)$ for some $\nu \in[0,1]$, then by (13) we have

$$
\begin{aligned}
& 2 r\left(\int_{\Omega} w f^{2} d \mu \int_{\Omega} w g^{2} d \mu-\left(\int_{\Omega} w f g d \mu\right)^{2}\right) \\
& \quad \leq \int_{\Omega} w f^{2} d \mu \int_{\Omega} w g^{2} d \mu-\int_{\Omega} w f^{2(1-\nu)} g^{2 \nu} d \mu \int_{\Omega} w f^{2 \nu} g^{2(1-\nu)} d \mu \\
& \quad \leq 2 R\left(\int_{\Omega} w f^{2} d \mu \int_{\Omega} w g^{2} d \mu-\left(\int_{\Omega} w f g d \mu\right)^{2}\right)
\end{aligned}
$$


If $f \geq 0, g>0, f^{2}, g^{2}, f^{2(1-\nu)} g^{2 \nu}, f^{2 \nu} g^{2(1-\nu)} \in L_{w}(\Omega, \mu)$ for some $\nu \in[0,1]$ and

$$
0<m \leq \frac{f}{g} \leq M<\infty
$$

for some constants $m, M$, then by (16)

$$
\begin{aligned}
& \int_{\Omega} w f^{2} d \mu \int_{\Omega} w g^{2} d \mu-\int_{\Omega} w f^{2(1-\nu)} g^{2 \nu} d \mu \int_{\Omega} w f^{2 \nu} g^{2(1-\nu)} d \mu \\
& \quad \leq R(M-m)^{2}\left(\int_{\Omega} w g^{2} d \mu\right)^{2},
\end{aligned}
$$

where $R=\max \{1-\nu, \nu\}$.

If $f, g \geq 0$ and $f^{p}, g^{q} \in L_{w}(\Omega, \mu)$ for $p, q>1$ with $\frac{1}{p}+\frac{1}{q}=1$, then by $(24)$

$$
\begin{aligned}
& 2 s(\left.\sqrt{\int_{\Omega} w f^{p} d \mu \int_{\Omega} w g^{q} d \mu}-\int_{\Omega} w f^{\frac{p}{2}} g^{\frac{q}{2}} d \mu\right) \\
& \times\left[\int_{\Omega} w f^{p} d \mu\right]^{\frac{1}{p}-\frac{1}{2}}\left[\int_{\Omega} w g^{q} d \mu\right]^{\frac{1}{q}-\frac{1}{2}} \\
& \leq\left[\int_{\Omega} w f^{p} d \mu\right]^{1 / p}\left[\int_{\Omega} w g^{q} d \mu\right]^{1 / q}-\int_{\Omega} w f g d \mu \\
& \leq 2 S\left(\sqrt{\left.\int_{\Omega} w f^{p} d \mu \int_{\Omega} w g^{q} d \mu-\int_{\Omega} w f^{\frac{p}{2}} g^{\frac{q}{2}} d \mu\right)}\right. \\
& \times\left[\int_{\Omega} w f^{p} d \mu\right]^{\frac{1}{p}-\frac{1}{2}}\left[\int_{\Omega} w g^{q} d \mu\right]^{\frac{1}{q}-\frac{1}{2}},
\end{aligned}
$$

where $s=\min \left\{\frac{1}{p}, \frac{1}{q}\right\}$ and $S=\max \left\{\frac{1}{p}, \frac{1}{q}\right\}$.

Let $f, g$ be $\mu$-measurable functions defined on $\Omega$ and

$$
0<m_{1} \leq f \leq M_{1}<\infty, 0<m_{2} \leq g \leq M_{2}<\infty \text { a.e. on } \Omega
$$

for some constants $m_{1}, m_{2}, M_{1}$ and $M_{2}$, then by (28)

$$
\begin{aligned}
0 \leq & {\left[\int_{\Omega} w f^{p} d \mu\right]^{1 / p}\left[\int_{\Omega} w g^{q} d \mu\right]^{1 / q}-\int_{\Omega} w f g d \mu } \\
\leq & S\left(\max \left\{\left(\frac{M_{1}}{m_{1}}\right)^{\frac{p}{2}},\left(\frac{M_{2}}{m_{2}}\right)^{\frac{q}{2}}\right\}-\min \left\{\left(\frac{m_{1}}{M_{1}}\right)^{\frac{p}{2}},\left(\frac{m_{2}}{M_{2}}\right)^{\frac{q}{2}}\right\}\right)^{2} \\
& \times\left[\int_{\Omega} w f^{p} d \mu\right]^{1 / p}\left[\int_{\Omega} w g^{q} d \mu\right]^{1 / q} .
\end{aligned}
$$


In particular, we have

$$
\begin{aligned}
0 \leq & {\left[\int_{\Omega} w f^{2} d \mu\right]^{1 / 2}\left[\int_{\Omega} w g^{2} d \mu\right]^{1 / 2}-\int_{\Omega} w f g d \mu } \\
\leq & S\left(\max \left\{\frac{M_{1}}{m_{1}}, \frac{M_{2}}{m_{2}}\right\}-\min \left\{\frac{m_{1}}{M_{1}}, \frac{m_{2}}{M_{2}}\right\}\right)^{2} \\
& \times\left[\int_{\Omega} w f^{2} d \mu\right]^{1 / 2}\left[\int_{\Omega} w g^{2} d \mu\right]^{1 / 2} .
\end{aligned}
$$

\section{Applications for real numbers}

We consider the $n$-tuples of positive numbers $a=\left(a_{1}, \ldots, a_{n}\right), b=\left(b_{1}, \ldots, b_{n}\right)$ and the probability distribution $p=\left(p_{1}, \ldots, p_{n}\right)$, i.e. $p_{i} \geq 0$ for any $i \in$ $\{1, \ldots, n\}$ with $\sum_{i=1}^{n} p_{i}=1$.

If we use the inequality (31) for the counting discrete measure, then we have

$$
\begin{aligned}
& 2 r\left(\sum_{i=1}^{n} p_{i} a_{i}^{2} \sum_{i=1}^{n} p_{i} b_{i}^{2}-\left(\sum_{i=1}^{n} p_{i} a_{i} b_{i}\right)^{2}\right) \\
& \leq \sum_{i=1}^{n} p_{i} a_{i}^{2} \sum_{i=1}^{n} p_{i} b_{i}^{2}-\sum_{i=1}^{n} p_{i} a_{i}^{2(1-\nu)} b_{i}^{2 \nu} \sum_{i=1}^{n} p_{i} a_{i}^{2 \nu} b_{i}^{2(1-\nu)} \\
& \quad \leq 2 R\left(\sum_{i=1}^{n} p_{i} a_{i}^{2} \sum_{i=1}^{n} p_{i} b_{i}^{2}-\left(\sum_{i=1}^{n} p_{i} a_{i} b_{i}\right)^{2}\right)
\end{aligned}
$$

where $\nu \in[0,1], r=\min \{1-\nu, \nu\}$ and $R=\max \{1-\nu, \nu\}$.

If there exists some constants $m, M$ such that

$$
0<m \leq \frac{a_{i}}{b_{i}} \leq M<\infty \text { for any } i \in\{1, \ldots, n\},
$$

then by (33) we have that

$$
\begin{aligned}
& \sum_{i=1}^{n} p_{i} a_{i}^{2} \sum_{i=1}^{n} p_{i} b_{i}^{2}-\sum_{i=1}^{n} p_{i} a_{i}^{2(1-\nu)} b_{i}^{2 \nu} \sum_{i=1}^{n} p_{i} a_{i}^{2 \nu} b_{i}^{2(1-\nu)} \\
& \quad \leq R(M-m)^{2}\left(\sum_{i=1}^{n} p_{i} b_{i}^{2}\right)^{2}
\end{aligned}
$$

where $R=\max \{1-\nu, \nu\}$. 
If $p, q>1$ with $\frac{1}{p}+\frac{1}{q}=1$, then by (34) we have

$$
\begin{aligned}
& \text { (40) } 2 s\left(\sqrt{\sum_{i=1}^{n} p_{i} a_{i}^{p} \sum_{i=1}^{n} p_{i} b_{i}^{q}}-\sum_{i=1}^{n} p_{i} a_{i}^{\frac{p}{2}} b_{i}^{\frac{q}{2}}\right)\left[\sum_{i=1}^{n} p_{i} a_{i}^{p}\right]^{\frac{1}{p}-\frac{1}{2}}\left[\sum_{i=1}^{n} p_{i} b_{i}^{q}\right]^{\frac{1}{q}-\frac{1}{2}} \\
& \leq\left[\sum_{i=1}^{n} p_{i} a_{i}^{p}\right]^{1 / p}\left[\sum_{i=1}^{n} p_{i} b_{i}^{q}\right]^{1 / q}-\sum_{i=1}^{n} p_{i} a_{i} b_{i} \\
& \leq 2 S\left(\sqrt{\sum_{i=1}^{n} p_{i} a_{i}^{p} \sum_{i=1}^{n} p_{i} b_{i}^{q}}-\sum_{i=1}^{n} p_{i} a_{i}^{\frac{p}{2}} b_{i}^{\frac{q}{2}}\right) \\
& \times\left[\sum_{i=1}^{n} p_{i} a_{i}^{p}\right]^{\frac{1}{p}-\frac{1}{2}}\left[\sum_{i=1}^{n} p_{i} b_{i}^{q}\right]^{\frac{1}{q}-\frac{1}{2}}
\end{aligned}
$$

where $s=\min \left\{\frac{1}{p}, \frac{1}{q}\right\}$ and $S=\max \left\{\frac{1}{p}, \frac{1}{q}\right\}$.

If for the constants $m_{1}, m_{2}, M_{1}$ and $M_{2}$ we have

$$
0<m_{1} \leq a_{i} \leq M_{1}<\infty, 0<m_{2} \leq b_{i} \leq M_{2}<\infty, \text { for any } i \in\{1, \ldots, n\}
$$

then by (36) we have

$$
\begin{aligned}
0 \leq & {\left[\sum_{i=1}^{n} p_{i} a_{i}^{p}\right]^{1 / p}\left[\sum_{i=1}^{n} p_{i} b_{i}^{q}\right]^{1 / q}-\sum_{i=1}^{n} p_{i} a_{i} b_{i} } \\
\leq & S\left(\max \left\{\left(\frac{M_{1}}{m_{1}}\right)^{\frac{p}{2}},\left(\frac{M_{2}}{m_{2}}\right)^{\frac{q}{2}}\right\}-\min \left\{\left(\frac{m_{1}}{M_{1}}\right)^{\frac{p}{2}},\left(\frac{m_{2}}{M_{2}}\right)^{\frac{q}{2}}\right\}\right)^{2} \\
& \times\left[\sum_{i=1}^{n} p_{i} a_{i}^{p}\right]^{1 / p}\left[\sum_{i=1}^{n} p_{i} b_{i}^{q}\right]^{1 / q} .
\end{aligned}
$$

In particular, we have

$$
\begin{aligned}
0 \leq & {\left[\sum_{i=1}^{n} p_{i} a_{i}^{2}\right]^{1 / 2}\left[\sum_{i=1}^{n} p_{i} b_{i}^{2}\right]^{1 / 2}-\sum_{i=1}^{n} p_{i} a_{i} b_{i} } \\
\leq & S\left(\max \left\{\frac{M_{1}}{m_{1}}, \frac{M_{2}}{m_{2}}\right\}-\min \left\{\frac{m_{1}}{M_{1}}, \frac{m_{2}}{M_{2}}\right\}\right)^{2} \\
& \times\left[\sum_{i=1}^{n} p_{i} a_{i}^{2}\right]^{1 / 2}\left[\sum_{i=1}^{n} p_{i} b_{i}^{2}\right]^{1 / 2} .
\end{aligned}
$$




\section{References}

[1] Andrica D., Badea C., Grüss' inequality for positive linear functionals, Periodica Math. Hung., 19(1998), 155-167.

[2] Beesack P.R., PeČArić J.E., On Jessen's inequality for convex functions, J. Math. Anal. Appl., 110(1985), 536-552.

[3] Callebaut D.K., Generalization of Cauchy-Schwarz inequality, J. Math. Anal. Appl., 12(1965), 491-494.

[4] Dragomir S.S., A refinement of Hadamard's inequality for isotonic linear functionals, Tamkang J. Math, (Taiwan), 24(1992), 101-106.

[5] Dragomir S.S., On a reverse of Jessen's inequality for isotonic linear functionals, J. Ineq. Pure \& Appl. Math., 2(3)(2001), Article 36, [On line: http://jipam.vu.edu.au/v2n3/047_01.html].

[6] Dragomir S.S., On the Jessen's inequality for isotonic linear functionals, Nonlinear Analysis Forum, 7(2)(2002), 139-151.

[7] Dragomir S.S., On the Lupaş-Beesack-Pečarić inequality for isotonic linear functionals, Nonlinear Funct. Anal. \& Appl., 7(2)(2002), 285-298.

[8] Dragomir S.S., Bounds for the normalized Jensen functional, Bull. Austral. Math. Soc., 74(3)(2006), 417-478.

[9] Dragomir S.S., Ionescu N.M., On some inequalities for convex-dominated functions, L'Anal. Num. Théor. L'Approx., 19(1) (1990), 21-27.

[10] Dragomir S.S., Pearce C.E.M., Selected Topics on Hermite-Hadamard Inequalities and Applications, RGMIA Monographs, Victoria University, 2000. [http://rgmia.vu.edu.au/monographs.html].

[11] Dragomir S.S., Pearce C.E.M., Pečarić J.E., On Jessen's and related inequalities for isotonic sublinear functionals, Acta. Sci. Math. (Szeged), 61(1995), 373-382.

[12] Kittaneh F., Manasrah Y., Improved Young and Heinz inequalities for matrix, J. Math. Anal. Appl., 361(2010), 262-269.

[13] Kittaneh F., Manasrah Y., Reverse Young and Heinz inequalities for matrices, Linear Multilinear Algebra, 59(2011), 1031-1037.

[14] LupAş A., A generalisation of Hadamard's inequalities for convex functions, Univ. Beograd. Elek. Fak., 577-579 (1976), 115-121.

[15] PeČArić J.E., On Jessen's inequality for convex functions (III), J. Math. Anal. Appl., 156(1991), 231-239.

[16] PeČarić J.E., BeEsack P.R., On Jessen's inequality for convex functions (II), J. Math. Anal. Appl., 156(1991), 231-239.

[17] Pečarić J.E., Dragomir S.S., A generalisation of Hadamard's inequality for isotonic linear functionals, Radovi Mat. (Sarjevo), 7(1991), 103-107.

[18] PeČarić J.E., Raşa I., On Jessen's inequality (Szeged), Acta. Sci. Math., 56(1992), 305-309.

[19] Specht W., Zer Theorie der elementaren Mittel, Math. Z., 74(1960), 91-98.

[20] Toader G., Dragomir S.S., Refinement of Jessen's inequality, Demonstratio Mathematica, 28(1995), 329-334.

[21] Tominaga M., Specht's ratio in the Young inequality, Sci. Math. Japon., $55(2002), 583-588$. 


\section{S.S. DRAGOMIR}

Mathematics, College of Engineering \& Science

VICTORIA UNIVERSITY

Melbourne City, MC 8001, Australia

OR

DST-NRF CENTRE OF EXCELlence

in the Mathematical and Statistical Sciences

School of Computer Science And Applied Mathematics

UNIVERSITY OF THE WITWATERSRAND

Private Bag 3, Johannesburg 2050, South Africa

e-mail: sever.dragomir@vu.edu.au

Received on 18.04.2017 and, in revised form, on 08.11.2017. 